\title{
Consumo alimentar de risco e proteção para as doenças crônicas não transmissíveis e sua associação com a gordura corporal: um estudo com funcionários da área de saúde de uma universidade pública de Recife (PE), Brasil
}

\author{
Risk and protection food consumption factors for chronic \\ non-communicable diseases and their association with body fat: \\ a study of employees in the health area of a public university \\ in Recife in the state of Pernambuco, Brazil
}

Edynara Cristiane de Castro Azevedo ${ }^{1}$

Fábia Morgana Rodrigues da Silva Dias ${ }^{1}$

Alcides da Silva Diniz ${ }^{1}$

Poliana Coelho Cabral ${ }^{1}$

${ }^{1}$ Programa de PósGraduação em Nutrição, Centro de Ciências da

Saúde, Universidade Federal de Pernambuco (UFPE) Av. Prof. Moraes Rego no 1235 , Cidade Universitária. 50.670-901 Recife PE Brasil.

edy_azevedo@hotmail.com
Abstract This article seeks to assess the consumption of risk and protection foods for chronic noncommunicable diseases and its association with body fat by health area workers in a public university in Recife in the state of Pernambuco. This cross-sectional study involved 267 adults. Two food groups were considered: risk and protection foods. Food consumption was assessed by a food frequency questionnaire with measurements converted to scores. The conceptual model considered sociodemographic, behavioral, and anthropometric variables. A high prevalence of overweight and low consumption of protection foods was detected. The average scores of risk and protection food consumption were similar in all variables analyzed, except for a higher consumption of protection foods observed in obese individuals $(p=0.000)$. The study highlights the complexity involved in the relation between food consumption, body fat, and chronic non-communicable diseases, indicating the need of future studies with more appropriate designs to provide input for future interventions in this population.

Key words Food consumption, Adults, Anthropometry, Chronic non-communicable diseases, Body fat
Resumo O objetivo deste artigo é avaliar o consumo de alimentos de risco e proteção para as doenças crônicas não transmissíveis e sua associação com a gordura corporal por funcionários da área de saúde de uma universidade pública da cidade de Recife, em Pernambuco. Estudo transversal, envolvendo 267 adultos. Foram constituídos 2 grupos de alimentos: Risco e proteção. O consumo alimentar foi avaliado por um questionário de frequência alimentar com mensuração convertida em escores. O modelo conceitual considerou variáveis sociodemográficas, comportamentais e antropométricas. Foi evidenciada uma elevada prevalência de excesso de peso e do baixo consumo de alimentos protetores. As medianas dos escores de consumo de alimentos de risco e proteção foram similares em todas as variáveis analisadas, exceto por um maior consumo de alimentos protetores observado nos individuos obesos $(p=0,000)$. O estudo aponta para a complexidade envolvida na relação entre consumo alimentar, gordura corporal e doenças crônicas não transmissiveis, indicando a necessidade de novos estudos com delineamentos mais apropriados, fornecendo subsídios para futuras intervenções nesta população.

Palavras-chave Consumo alimentar, Adultos, Antropometria, Doenças crônicas não transmissíveis, Gordura corporal 


\section{Introdução}

As doenças crônicas não transmissíveis (DCNT) são atualmente as principais causas de morte no Brasil e no mundo. Estudos mostram que hábitos alimentares inadequados, dentre outros $\mathrm{fa}$ tores, têm sido associados às DCNT, como o diabetes melittus, as doenças cardiovasculares, alguns tipos de câncer e a obesidade ${ }^{1}$.

O padrão alimentar da população brasileira tem sofrido mudanças, caracterizadas pelo aumento da ingestão de alimentos com alta densidade energética, pobres em fibras ${ }^{2}$ e ricos em gordura saturada, gordura trans e açúcares simples, associados à inatividade física, tabagismo e consumo excessivo de álcool, os quais têm sido identificados como preditores independentes dessas enfermidades ${ }^{1}$.

O aumento significativo na prevalência de excesso de peso e obesidade tem sido considerado como um dos principais fatores de risco para as DCNT, pois se sabe que o risco destas aumenta diretamente com o grau de excesso de peso e acúmulo de gordura na região abdominal ${ }^{1}$. Esses achados são confirmados pela Pesquisa de Orçamentos Familiares (POF 2008-2009) ${ }^{3}$, que constatou uma prevalência de $50 \%$ de excesso de peso na população adulta brasileira e baixo consumo de alimentos ricos em fibras, como frutas, verduras e leguminosas e alto consumo de alimentos ricos em gordura saturada, açúcar e sal. Dados recentes ${ }^{4}$ mostram que menos de $1 / 4$ da população possui consumo recomendado de frutas e hortaliças, o que reflete a baixa qualidade da dieta do brasileiro.

Estudos têm analisado a associação entre o risco de doenças crônicas e o consumo de determinados alimentos e nutrientes ${ }^{5,6}$. Porém, a OMS sugere que a avaliação do consumo alimentar das populações estaria mais bem representada pelo padrão alimentar, considerando que os indivíduos não consomem nutrientes nem alimentos isoladamente ${ }^{7}$. Nesse sentido, é crescente o interesse nessa linha de investigação sobre o consumo de grupos de alimentos considerados definidores de padrões alimentares saudáveis e não saudáveis. A questão que se coloca seria até que ponto a pesquisa empírica tem abordado a questão do padrão alimentar como variável preditiva da gordura corporal. Portanto, o presente estudo tem como objetivo avaliar o consumo alimentar de risco e proteção para as DCNT e sua associação com a gordura corporal de funcionários da área de saúde de uma universidade pública da cidade do Recife, em Pernambuco.

\section{Métodos}

O estudo é do tipo transversal, envolvendo funcionários acima de 20 anos, de ambos os sexos, provenientes da área de saúde da Universidade Federal de Pernambuco, realizado no período de abril a setembro de 2010 .

O tamanho amostral foi determinado tomando-se como base a estimativa da frequência adequada do consumo de frutas, hortaliças e leguminosas, observadas no estudo piloto. Essa estimativa foi possível, pois dentre os dados coletados também constavam perguntas sobre a quantidade de porções de alimentos consumidos diariamente referentes a cada grupo da pirâmide alimentar para adultos, adaptada de Philipi et al. ${ }^{8}$. Desse modo, adotando-se uma frequência de consumo de $20 \%$, com uma margem de erro aceitável de 4\%, uma confiabilidade de 95\% e uma população de cerca de 634 funcionários ( 425 docentes e 209 técnicos), o tamanho amostral mínimo foi de 240. Para corrigir eventuais perdas, esse valor foi corrigido em 10\% [100/(100-10)], totalizando uma amostra de 267 indivíduos.

Os indivíduos foram selecionados mediante um processo de adesão espontânea, a partir da divulgação do estudo entre professores e funcionários dos cursos do Centro de Ciências da Saúde (CCS) da UFPE. Foram utilizados os seguintes critérios de elegibilidade: ser do quadro efetivo, não ser portador de deficiência física que impedisse a avaliação antropométrica e não ser gestante ou lactante. Os dados referentes ao consumo alimentar foram coletados por meio de um questionário de frequência alimentar (QFA) do tipo qualitativo, sendo desenvolvido e validado por Furlan-Viebig e Pastor-Valero' ${ }^{9}$, para o estudo da dieta e DCNT. O questionário proposto por esses autores é composto por 98 itens, sendo adotadas as seguintes categorias de frequência de consumo (FC): Nunca (FC1), $\leq 1$ vez por mês (FC2), 1 vez por semana (FC3), 2-4 vezes por semana (FC4), 1 vez ao dia (FC5), e 2 ou mais vezes ao dia (FC6). Para que a FC de cada alimento pudesse ser tratada como FC anual, foi atribuído um peso (S) a cada categoria de FC.

Foi definido como peso (S) máximo o valor igual a 1 para a frequência de 2 ou mais vezes ao dia (FC6). Os demais pesos foram obtidos de acordo com a seguinte equação: $S n=(1 / 365) \mathrm{x}$ $[(\mathrm{a}+\mathrm{b}) / 2]$ sendo que $a$ e $b$ representam o número de dias da frequência. Assim, para cada indivíduo, foi obtido o cálculo do escore de frequência de consumo de alimentos correspondente a dois grupos (Grupo I e Grupo II). O Grupo I foi for- 
mado pelos alimentos considerados de risco para as DCNT e o Grupo II pelos protetores.

- Grupo I: Leite integral e produtos lácteos integrais (iogurte integral, queijos amarelos, creme de leite); gorduras de origem animal (manteiga); gorduras de origem vegetal (margarinas); alimentos fritos e fast food (salgadinhos de bar, coxinha, empada, pizza, sanduíche, McDonalds); carnes com gordura, fritas e/ou com pele (aves, bovina, suína, fígado, vísceras de frango e de boi); produtos derivados (embutidos, charque, mortadela, presunto, linguiça, salsicha, preparações à base de carnes), refrigerantes, bebidas alcoólicas (cerveja, vinho, pinga, uísque), sucos artificiais, açúcares e sobremesas (balas, doces, mel, rapadura, pudim, manjar, doces, sorvetes, bolos).

- Grupo II: Leite desnatado e produtos lácteos desnatados (iogurte, queijos brancos), frutas e sucos naturais, água de coco, verduras e legumes (crus e cozidos), leguminosas (feijão mulatinho, carioca, preto, verde e macassa), cereais e derivados (arroz, macarrão, pães, biscoitos salgados, milho, aveia), raízes e tubérculos (macaxeira, inhame, farinha de mandioca, batata doce e inglesa), carnes cozidas e/ou sem pele (aves e bovinas), peixes e frutos do mar.

O consumo alimentar também foi avaliado em função de variáveis sociodemográficas, comportamentais (estilo de vida) e antropométricas. Dentre as variáveis sociodemográficas, consideraram-se: sexo, idade em anos no momento da entrevista, categorizada em duas faixas etárias (20 a 49 e $\geq 50$ anos); e escolaridade estratificada em $\leq$ ensino fundamental, $\leq$ ensino médio e ensino superior. Os aspectos referentes ao estilo de vida foram: hábito de fumar, sendo considerados fumantes, não fumantes e ex-fumantes; consumo de álcool, agrupado em bebe e não bebe e atividade física dicotomizada em pratica exercício físico (sim ou não), se sim, qual e em que frequência.

Para a identificação de sobrepeso, obesidade, obesidade abdominal e percentual de gordura corporal de risco relacionado à obesidade, foram utilizados o índice de massa corporal (IMC), a circunferência da cintura (CC) e a bioimpedância elétrica (BIA). As medidas antropométricas foram aferidas em duplicata pelo mesmo avaliador e desprezadas quando o erro de aferição entre elas foi maior que $100 \mathrm{~g}$ para peso, 0,5 $\mathrm{cm}$ para altura e $0,1 \mathrm{~cm}$ para circunferência da cintura. O valor resultante das aferições foi a média entre elas.

Para a determinação do peso corporal e estatura dos funcionários foi utilizada uma balança eletrônica digital Plena, capacidade $150 \mathrm{Kg}$ com divisão de $100 \mathrm{~g}$ e um estadiômetro portátil (Ghrum Polar Manufacture, Suíça) com extensão de 2,00m, dividido em centímetros e subdividido em milímetros. Tanto o peso quanto a altura foram mensurados segundo técnicas preconizadas por Lohman et al..$^{10} \mathrm{e}$ serviram de base para o cálculo do IMC, sendo que a classificação utilizada foi a proposta pela OMS ${ }^{11}$.

Com o objetivo de identificar a obesidade abdominal, foi utilizada a CC, aferida com uma fita métrica não extensível, Sanny (American Medical do Brasil Ltda., São Bernardo do Campo, Brasil), com precisão de $1 \mathrm{~mm}$, no ponto médio entre última costela e a crista do ilíaco. Os pontos de corte adotados foram os preconizados pela $\mathrm{OMS}^{12}$, de acordo com o risco de complicações metabólicas associadas à obesidade: risco elevado para mulheres $(\mathrm{CC} \geq 80 \mathrm{~cm})$ e para homens $(C C \geq 94 \mathrm{~cm})$.

As medidas de bioimpedância elétrica (BIA) foram realizadas com o aparelho Maltron BF906 (Maltron, Reino Unido), com uma frequência de $50 \mathrm{~Hz}$ em corrente alternada de quatro eletrodos. O aparelho fornecia o percentual de gordura diretamente através de equações já programadas pelos fabricantes no próprio instrumento. As medidas foram feitas com o indivíduo deitado sobre uma superfície não condutora (colchonete), na posição supina, com pernas e braços abduzidos a $45^{\circ}$, sem portar brincos, relógio, anéis e objetos metálicos. Os participantes foram orientados a seguir alguns procedimentos prévios, visando assegurar a acurácia das aferições: jejum absoluto de 4 horas; não realizar exercícios físicos extenuantes 12 horas antes de teste; não ingerir bebidas alcoólicas 48 horas antes do teste; não ingerir medicamentos que influenciem no equilíbrio hidroeletrolítico a menos de 7 dias do teste e urinar pelo menos 30 minutos antes do teste $^{13}$. Para indicar níveis de gordura corporal de risco relacionados à obesidade, foram utilizados os valores de $28 \%$ para homens e $40 \%$ para mulheres, pontos de cortes recomendados por Gallagher et al. ${ }^{14}$.

O protocolo de pesquisa foi submetido à apreciação e aprovado pelo comitê de ética da Universidade Federal de Pernambuco. Os funcionários que concordaram em participar da pesquisa receberam todas as informações sobre o estudo e assinaram o termo de consentimento livre e esclarecido.

A construção do banco de dados foi realizada no programa Epi-info versão 6.04 (CDC/ WHO, Atlanta, GE, USA) com dupla entrada de 
informações e verificadas com o módulo VALIDATE, para checar a consistência e a validação das mesmas. As análises estatísticas foram realizadas no SPSS 7.5 para Windows (SPSS Inc., Chicago, IL, USA).

Os escores de frequência de consumo alimentar, em virtude de se tratar de variáveis em escala ordinal, foram descritos sob a forma de mediana e Intervalo Interquartílico (IQ). A associação entre o consumo alimentar e as variáveis explicativas foi avaliada pelos testes "U" de Mann Whitney (duas medianas) e Kruskal Wallis (mais de duas medianas), empregando-se o teste "U" de Mann Whitney a posteriori. Na validação das associações investigadas foi adotado o valor de $\mathrm{p}<$ 0,05 .

\section{Resultados}

Foram analisados dados de 267 funcionários, sendo 135 do sexo masculino (50,6\%), com média de idade 43,5 $\pm 11,5$ anos. Observou-se que $27,4 \%$ dos homens e $8,3 \%$ das mulheres possuíam grau de instrução até o ensino fundamental, por outro lado, $28,9 \%$ e $57,6 \%$, respectivamente, haviam cursado o ensino superior e, dentre estes, muitos eram pós-graduados $(\mathrm{p}=0,000)(\mathrm{Ta}-$ bela 1).

Na Tabela 1 também se encontra descrita a frequência de alterações nutricionais segundo o IMC e a CC. Na aferição de tais medidas antropométricas ocorreu a perda de alguns funcionários, 23 se recusaram a aferir a CC e destes, 6 também não permitiram a aferição do peso. Foi evidenciado que aproximadamente $60,0 \%$ da amostra estudada apresentaram excesso de peso, dentre os quais, cerca de 20,0\% eram obesos, não sendo encontrado diferencial estatisticamente significante entre os sexos. Apenas duas pessoas estavam abaixo do peso e, por isso, elas foram agrupadas junto às de peso normal. Quanto à CC, $56,3 \%$ dos homens e $81,6 \%$ das mulheres foram classificados na faixa de risco elevado $(p=0,000)$. Não foi possível aferir a gordura corporal pela BIA em 24 funcionários, alguns se recusaram ( $\mathrm{n}$ $=9)$ e outros $(n=15)$ não seguiram as recomendações feitas anteriormente quanto aos cuidados pré-exame. Nesta aferição cerca de $30,0 \%$ dos avaliados apresentaram percentual de gordura corporal na faixa de risco relacionado à obesidade, não sendo evidenciado diferencial estatisticamente significante entre os sexos.

No que se refere ao estilo de vida, os homens praticavam mais exercício físico (55,6\% x 37,1\% $\mathrm{p}=0,004)$, bebiam mais $(71,1 \% \times 53,8 \% \mathrm{p}=$ $0,005)$ e fumavam mais que as mulheres $(17,8 \%$ $\mathrm{x} 7,6 \% \mathrm{p}=0,020$ ) (Tabela 1). Em relação ao padrão alimentar, 19,9\% dos funcionários referiram estar em dieta para perda ponderal. A proporção de portadores de sobrepeso e obesidade que estavam em dieta foi de $27,0 \%$ e $29,4 \%$, respectivamente.

$\mathrm{Na}$ análise da Figura 1, observa-se que a mediana de escores de consumo mensal de alimentos de risco (Grupo I) foi similar $(\mathrm{p}=0,837)$ à encontrada para o consumo de alimentos protetores (Grupo II). No entanto, a dispersão no Grupo II, avaliada pelo coeficiente de variação interquartílico (CVI) foi bem superior à evidenciada no Grupo I (37,8\% contra 29,7\%).

Na Tabela 2 encontram-se descritas as medianas e os intervalos interquartílicos dos escores de consumo alimentar segundo características socioeconômicas, demográficas, comportamentais e antropométricas. Dessas variáveis, apenas os funcionários com IMC $\geq 30,0 \mathrm{Kg} / \mathrm{m}^{2}$ (obesos) e com percentual de gordura corporal na faixa de risco associada à obesidade ( $\geq 28 \%$ homens e $\geq 40 \%$ mulheres) apresentaram maiores medianas de escores de consumo de alimentos protetores $(p=0,000)$, quando comparados aos eutróficos e aqueles com sobrepeso. Para as demais variáveis, houve similaridade na distribuição das medianas dos escores de consumo alimentar entre os dois grupos (I e II).

\section{Discussão}

Os padrões alimentares têm sido cada vez mais utilizados em estudos que objetivam conhecer o perfil de consumo alimentar das populações e sua associação com as DCNT. Tal abordagem qualitativa apresenta vantagens em relação à análise quantitativa de alimentos ou nutrientes, tendo em vista que os indivíduos não os consomem de forma isolada, mas em refeições, contendo vários alimentos, com inúmeras combinações de nutrientes que nesse caso são mais bem avaliados quando o estudo é realizado focando os padrões alimentares ${ }^{15}$.

Sabe-se que os padrões alimentares podem ser bem diferentes entre as diversas populações e por esse motivo de difícil generalização, sendo resultado da complexa interação entre diversos fatores como ambientais, demográficos, sociais, econômicos e culturais ${ }^{16}$. Além disso, as escolhas alimentares podem se modificar ao longo do tempo, de acordo com a disponibilidade dos 
Tabela 1. Características sociodemográficas, comportamentais e antropométricas de funcionários da área de saúde da Universidade Federal de Pernambuco (UFPE) - Recife (2011).

\begin{tabular}{|c|c|c|c|c|c|c|c|}
\hline \multirow{2}{*}{ Variáveis } & \multicolumn{3}{|c|}{ Masculino } & \multicolumn{3}{|c|}{ Feminino } & \multirow{2}{*}{$\mathbf{p}^{\mathrm{c}}$} \\
\hline & $\mathbf{N}$ & $\%$ & IC $95 \%$ & $\mathbf{N}$ & $\%$ & IC $95 \%$ & \\
\hline Grau de instrução & & & & & & & 0,000 \\
\hline$\leq$ ensino fundamental & 37 & 27,4 & $(20,2-35,9)$ & 11 & 8,3 & $(4,4-14,8)$ & \\
\hline$\leq$ ensino médio & 59 & 43,7 & $(35,3-52,5)$ & 45 & 34,1 & $(26,2-42,9)$ & \\
\hline ensino superior & 39 & 28,9 & $(21,6-37,4)$ & 76 & 57,6 & $(48,7-66,0)$ & \\
\hline Total & 135 & 100,0 & & 132 & 100,0 & & \\
\hline Faixa etária (anos) & & & & & & & 0,004 \\
\hline $20-49$ & 101 & 74,8 & $(66,5-81,7)$ & 76 & 57,6 & $(48,7-66,0)$ & \\
\hline$\geq 50$ & 34 & 25,2 & $(18,3-33,5)$ & 56 & 42,4 & $(34,0-51,3)$ & \\
\hline Total & 135 & 100,0 & & 132 & 100,0 & & \\
\hline $\operatorname{IMC}\left(\mathrm{kg} / \mathrm{m}^{2}\right)$ & & & & & & & 0,656 \\
\hline Eutrofia & 47 & 35,6 & $(27,6-44,5)$ & 53 & 41,1 & $(32,6-50,1)$ & \\
\hline Sobrepeso & 57 & 43,2 & $(34,7-52,1)$ & 50 & 38,8 & $(30,4-47,8)$ & \\
\hline Obesidade & 28 & 21,2 & $(14,8-29,4)$ & 26 & 20,2 & $(13,8-28,3)$ & \\
\hline Total & 132 & 100,0 & & 129 & 100,0 & & \\
\hline $\mathrm{CC}(\text { risco elevado })^{\mathrm{a}}$ & & & & & & & 0,000 \\
\hline Sim & 67 & 56,3 & $(46,9-65,3)$ & 102 & 81,6 & $(73,5-87,4)$ & \\
\hline Não & 52 & 43,7 & $(34,7-53,1)$ & 23 & 18,4 & $(12,3-26,5)$ & \\
\hline Total & 119 & 100,0 & & 125 & 100,0 & & \\
\hline Gordura corporal (BIA) & & & & & & & 0,812 \\
\hline Com risco ${ }^{*}$ & 38 & 31,1 & $(23,2-40,2)$ & 35 & 28,9 & $(21,2-37,9)$ & \\
\hline Sem risco ${ }^{* *}$ & 84 & 68,9 & $(59,7-76,7)$ & 86 & 71,1 & $(62,0-78,7)$ & \\
\hline Total & 122 & 100,0 & & 121 & 100,0 & & \\
\hline Exercício físico ${ }^{\mathrm{b}}$ & & & & & & & 0,004 \\
\hline Sim & 75 & 55,6 & $(46,8-64,0)$ & 49 & 37,1 & $(29,0-46,0)$ & \\
\hline Não & 60 & 44,4 & $(36,0-53,2)$ & 83 & 62,9 & $(54,0-71,0)$ & \\
\hline Total & 135 & 100,0 & & 132 & 100,0 & & \\
\hline Fuma & & & & & & & 0,020 \\
\hline $\operatorname{Sim}$ & 24 & 17,8 & $(11,9-25,5)$ & 10 & 7,6 & $(3,9-13,8)$ & \\
\hline Não e ex-fumante & 111 & 82,2 & $(74,5-88,1)$ & 122 & 92,4 & $(86,2-96,1)$ & \\
\hline Total & 135 & 100,0 & & 132 & 100,0 & & \\
\hline Bebe & & & & & & & 0,005 \\
\hline Sim & 96 & 71,1 & $(62,6-78,4)$ & 71 & 53,8 & $(44,9-62,4)$ & \\
\hline Não & 39 & 28,9 & $(21,6-37,4)$ & 61 & 46,2 & $(37,6-55,1)$ & \\
\hline Total & 135 & 100,0 & & 132 & 100,0 & & \\
\hline
\end{tabular}

Nota: O número total de participantes em cada variável é diferente por causa do número de respondentes. ${ }^{a}$ Circunferência da cintura (CC) risco elevado $>102 \mathrm{~cm}$ homens e $>88 \mathrm{~cm}$ mulheres. ${ }^{*}$ Com risco $\geq 28 \%$ homens e $\geq 40 \%$ mulheres; ${ }^{* *}$ Sem risco

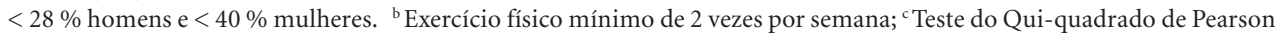

alimentos em cada região ${ }^{17}$. Apesar dessas limitações, tais padrões parecem refletir de forma mais apropriada a prática alimentar da população em estudo ${ }^{7}$. Dessa forma, podem subsidiar o planejamento das ações de saúde, possibilitando o estabelecimento de padrões regionais, úteis para estudos comparativos com outros grupos populacionais.

Deve-se salientar que os dados obtidos neste estudo provêm de funcionários da área de saúde de uma universidade pública do Nordeste brasileiro e, por conseguinte apresentam certa especi- ficidade. Os resultados encontrados, com $28,9 \%$ dos homens e $57,6 \%$ das mulheres tendo cursado ensino superior retratam um subgrupo populacional que, em principio, teriam um melhor conhecimento sobre os riscos de uma alimentação inadequada.

No entanto, as elevadas prevalências de sobrepeso e obesidade, em ambos os sexos, encontradas neste estudo, seguem a tendência preocupante do aumento do sobrepeso e obesidade observada no Brasil e em Pernambuco ao longo dos anos. Ou seja, os dados obtidos estão em 


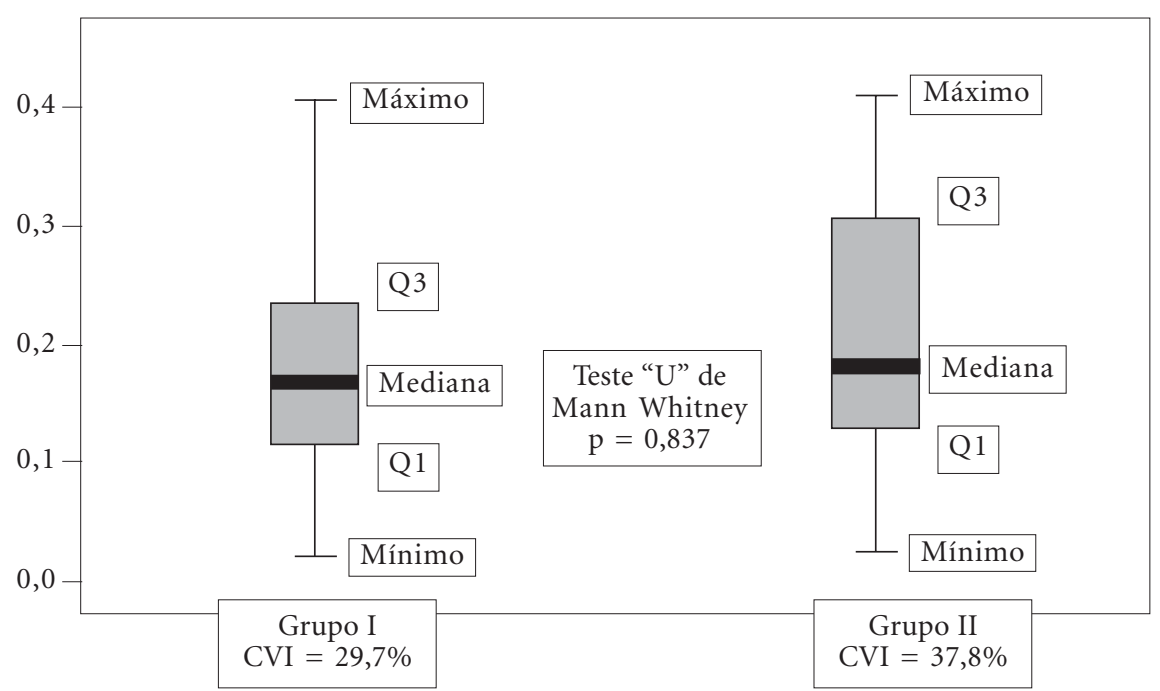

Figura 1. Distribuição dos escores (mediana, Q1, Q3, mínimo, máximo e coeficiente de variação interquartílico-CVI) do consumo de alimentos de risco para as DCNT (Grupo I) e de alimentos protetores (Grupo II) de funcionários de ambos os sexos da área de saúde da Universidade Federal de Pernambuco (UFPE) - Recife (2011).

concordância com os achados do último Sistema de Vigilância de Fatores de Risco e Proteção para Doenças Crônicas por inquérito telefônico $(\text { VIGITEL) })^{4}$ realizado em 2011, no qual 48,5\% da população adulta apresentaram excesso de peso, assemelhando-se também com os dados obtidos na última Pesquisa de Orçamentos Familiares (POF 2008/2009) $)^{3}$, no qual o excesso de peso foi observado em $50,1 \%$ dos homens e $48,1 \%$ das mulheres. Também, segundo dados obtidos na III Pesquisa Estadual de Saúde e Nutrição (III PESN 2006 $)^{18}$, quase metade da população adulta pernambucana apresentou excesso de peso.

Com relação à obesidade abdominal, importante fator de risco cardiovascular, mais mulheres $(81,6 \%)$ do que homens $(56,3 \%)$ possuíam risco elevado segundo a CC. No entanto, o acúmulo de gordura abdominal na mulher é mais de tecido adiposo subcutâneo (menor risco) e no homem o acúmulo maior é de gordura visceral (maior risco). Apesar disso, é importante enfatizar que o fato de o grupo avaliado estar exposto ao excesso de peso e de gordura abdominal, o coloca em condição de maior risco para as doenças cardiovasculares (DCV), independentemente do sexo.

Segundo Fornes et al. ${ }^{19}$, os escores alimentares refletem a qualidade da dieta, em que uma escala crescente nessa pontuação significaria um maior consumo de alimentos reconhecidos como de risco ou proteção para as DCV. No presente estudo, em relação aos escores de consumo alimentar de risco e proteção, não foi evidenciado diferencial entre os sexos, resultado que discorda com os achados de estudos prévios ${ }^{20-22}$, bem como, com aqueles encontrados no último VIGITEL, em $2011^{4}$, no qual as mulheres apresentaram maior consumo regular de frutas e hortaliças, em relação aos homens $(35,4 \%$ versus $25,6 \%$ ), mostrando uma aparente maior preocupação delas com a alimentação e com a saúde.

De fato, estudo realizado por Johnson et al. ${ }^{23}$ mostrou que as mulheres são mais preocupadas com o peso do que os homens, e tendem a recorrer a estratégias dietéticas quando querem uma perda ponderal, aumentando o consumo de verduras e frutas ${ }^{24}$ ou diminuindo a ingestão de proteínas e carboidratos ${ }^{25}$. Os homens, por outro lado, tendem a recorrer ao exercício físico para manter a boa forma, fato evidenciado neste estudo, com os homens exercendo mais essa prática que as mulheres. Desse modo, esperava-se que as mulheres ao praticar menos atividade física, utilizassem de outros meios, principalmente o dietético, para perda de peso e melhora da qualidade de vida, aumentando o consumo de alimentos como frutas, legumes e verduras. Esse resultado confirma a complexidade da obesidade e outras DCNT, pois embora fatores como hábitos alimentares e sedentarismo estejam as- 
Tabela 2. Medianas e intervalos interquartílicos dos escores de consumo alimentar segundo variáveis sociodemográficas, comportamentais e antropométricas em funcionários da área de saúde da Universidade Federal de Pernambuco (UFPE) - Recife (2011).

\begin{tabular}{|c|c|c|c|c|}
\hline \multirow{2}{*}{ Variável } & \multicolumn{4}{|c|}{ Grupo de alimentos } \\
\hline & \multicolumn{2}{|c|}{ Grupo I } & \multicolumn{2}{|c|}{ Grupo II } \\
\hline \multicolumn{5}{|l|}{ Sexo } \\
\hline Masculino & 0,1730 & $0,1130-0,2725$ & 0,1600 & $0,0965-0,2190$ \\
\hline Feminino & 0,1400 & $0,0750-0,2190$ & 0,1545 & $0,0965-0,2683$ \\
\hline p- valor & 0,227 & & 0,333 & \\
\hline \multicolumn{5}{|l|}{ Faixa etária (anos) } \\
\hline $20-49$ & 0,1780 & $0,1020-0,2450$ & 0,1495 & $0,0603-0,2518$ \\
\hline$\geq 50$ & 0,1500 & $0,0620-0,2160$ & 0,1890 & $0,0818-0,3195$ \\
\hline$\overline{\mathrm{p}}$-valor ${ }^{*}$ & 0,399 & & 0,128 & \\
\hline \multicolumn{5}{|l|}{ Bebe } \\
\hline Sim & 0,1760 & $0,1050-0,2510$ & 0,1510 & $0,0700-0,2640$ \\
\hline Não & 0,1190 & $0,0675-0,1980$ & 0,1480 & $0,0655-0,2695$ \\
\hline p-valor ${ }^{*}$ & 0,208 & & 0,586 & \\
\hline \multicolumn{5}{|l|}{ Instrução } \\
\hline$\leq$ ensino fundamental & 0,1430 & $0,0645-0,2225$ & 0,1375 & $0,0715-0,2718$ \\
\hline$\leq$ ensino médio & 0,1760 & $0,1175-0,2550$ & 0,1520 & $0,0785-0,2613$ \\
\hline ensino superior & 0,1250 & $0,0655-0,2350$ & 0,1465 & $0,0620-0,3005$ \\
\hline p-valor ${ }^{* *}$ & 0,493 & & 0,848 & \\
\hline \multicolumn{5}{|l|}{ IMC (kg/m2) } \\
\hline Eutrofia & 0,1670 & $0,0875-0,2415$ & $0,1476^{\mathrm{a}}$ & $0,0683-0,2723$ \\
\hline Sobrepeso & 0,1370 & $0,0715-0,2075$ & $0,1465^{\mathrm{a}}$ & $0,0683-0,2785$ \\
\hline Obesidade & 0,2020 & $0,1050-0,2680$ & $0,1790^{\mathrm{b}}$ & $0,0940-0,3160$ \\
\hline p-valor ${ }^{* *}$ & 0,268 & & 0,000 & \\
\hline \multicolumn{5}{|l|}{ CC $(\text { risco elevado })^{\#}$} \\
\hline $\operatorname{Sim}$ & 0,1530 & $0,0840-0,2270$ & 0,1520 & $0,0830-0,2898$ \\
\hline Não & 0,1630 & $0,0580-0,2215$ & 0,1565 & $0,0718-0,2748$ \\
\hline p-valor ${ }^{*}$ & 0,860 & & 0,736 & \\
\hline \multicolumn{5}{|l|}{ Gordura corporal (BIA)= } \\
\hline Com risco & 0,2234 & $0,1147-0,2953$ & $0,1861^{\mathrm{a}}$ & $0,1013-0,3327$ \\
\hline Sem risco & 0,1512 & $0,0937-0,2445$ & $0,1466^{\mathrm{b}}$ & $0,064-0,2764$ \\
\hline p-valor ${ }^{*}$ & 0,566 & & 0,000 & \\
\hline \multicolumn{5}{|l|}{ Exercício físico } \\
\hline Sim & 0,1540 & $0,0845-0,2385$ & 0,1825 & $0,0900-0,2910$ \\
\hline Não & 0,1600 & $0,0945-0,2270$ & 0,1370 & $0,0615-0,2595$ \\
\hline p-valor ${ }^{*}$ & 0,930 & & 0,075 & \\
\hline \multicolumn{5}{|l|}{ Fuma } \\
\hline Sim & 0,2350 & $0,1295-0,2690$ & 0,1600 & $0,1023-0,2610$ \\
\hline Não & 0,1550 & $0,0855-0,2240$ & 0,1550 & $0,0740-0,2455$ \\
\hline p-valor ${ }^{*}$ & 0,105 & & 0,478 & \\
\hline
\end{tabular}

\# Circunferência da cintura (CC) risco elevado $>102 \mathrm{~cm}$ homens e $>88 \mathrm{~cm}$ mulheres; " Com risco $\geq 28 \%$ homens e $\geq 40 \%$ mulheres e Sem risco $<28 \%$ homens e $<40 \%$ mulheres; • Exercício físico mínimo de 2 vezes por semana; "Teste "U” de Mann Whitney; ; Teste de Krukal Wallis. Teste a posteriori: Teste "U" de Mann Whitney; a,b Letras diferentes significam diferenças estatísticas entre as categorias.

sociados a essa patologias, a mudança do estilo de vida requer uma abordagem global, devendose considerar outros fatores que possam estar agindo nessa dinâmica ${ }^{26}$. Os resultados deste estudo assemelham-se com o realizado com adultos do estado de Pernambuco ${ }^{27}$, onde também não foram encontradas diferenças entre os escores de consumo alimentar por sexo.

Em relação às variáveis analisadas, apenas o consumo de alimentos protetores apresentou associação positiva com o estado nutricional, sendo os maiores escores de consumo desses ali- 
mentos observados entre os indivíduos classificados como obesos pelo IMC e BIA. Esses resultados contrariam os evidenciados por Castanho et $\mathrm{al}^{20}$, que ao analisarem o consumo de frutas, legumes e verduras em adultos e sua relação com a síndrome metabólica (SM), encontraram que o consumo adequado de frutas apresentou efeito protetor para a obesidade abdominal, hipertrigliceridemia e presença de SM.

Porém, os achados observados na nossa casuística assemelham-se aos encontrados em estudo realizado por Perozzo et al. ${ }^{15}$, os quais, ao analisarem a associação dos padrões alimentares com obesidade geral em mulheres adultas do Rio Grande do Sul, encontraram 5 tipos de padrões alimentares, através de análise fatorial; enquanto o consumo do "Padrão - Vegetais" apresentou associação direta com a obesidade, fato também observado em estudo de Sichieri et al. ${ }^{28}$. Resultados similares foram também relatados por Gimeno et al. ${ }^{17}$, ao analisarem os fatores associados ao padrão alimentar entre os participantes de um estudo populacional, em Ribeirão Preto, estado de São Paulo, em que encontraram 4 padrões alimentares e observaram maior frequência de indivíduos com padrão "saudável" entre aqueles com excesso de peso.

Esses resultados poderiam ter pelo menos duas explicações: seria plausível supor que esses indivíduos já estariam em tratamento, com dieta para perda ponderal e, portanto, apresentando um padrão alimentar saudável, configurando um quadro de causalidade reversa. Esse tipo de viés de difícil controle seria uma das limitações do delineamento transversal para o estudo de associações. De fato, $29,4 \%$ dos obesos referiram estar em dieta no momento da entrevista. Uma segunda explicação estaria relacionada à ocorrência de distorções no relato de alguns indivíduos com excesso de peso, superestimando o consumo de alimentos considerados saudáveis. Em relação ao consumo de alimentos protetores, os resultados encontrados por Mondini et al. ${ }^{21}$ assemelham-se aos observados no presente trabalho, não encontrando-se associação entre o consumo de frutas, legumes e hortaliças e a prática de atividade física. Esses resultados contrariam os achados de Neutzling et al. ${ }^{22}$, em que $\mathrm{a}$ atividade física esteve associada à maior frequ- ência de consumo destes alimentos. Já em relação à idade, nossos resultados contrariam os encontrados também por Neutzling et al..$^{22}$ e os de Lins et al. ${ }^{29}$, que observaram maiores frequências de consumo de frutas, legumes e hortaliças em indivíduos de maior idade.

A falta de estandardização para a definição dos padrões de consumo alimentar dificulta a comparação dos resultados obtidos nas diferentes localidades. Dessa forma, é necessário conhecer melhor a validade e a confiabilidade dos padrões alimentares, pois em métodos de obtenção a posteriori (análise fatorial ou análise de agrupamento) o investigador toma decisões que podem afetar o número, os tipos de padrões e sua capacidade de predizer doenças com longo período de risco em populações ${ }^{30}$. Um exemplo seria o estudo de Neumann et al. ${ }^{31}$, no qual alimentos considerados de risco para DCNT fizeram parte de diferentes padrões. Bem como, no estudo de Perozzo et al. ${ }^{15}$, em que alimentos considerados de proteção (vegetais e frutas) compuseram padrões de consumo diferentes.

Uma limitação do nosso estudo diz respeito ao fato de ter sido utilizado um desenho transversal, pois sabe-se que este tipo de delineamento não é o mais apropriado para investigar as associações entre doenças crônicas e dieta. No entanto, estudos transversais de base populacional com amostras representativas têm grande relevância, por serem alternativas rápidas e baratas e muito úteis ao promoverem ações educativas e de promoção de hábitos alimentares saudáveis.

\section{Conclusão}

Embora o consumo de alimentos protetores, como frutas, legumes e verduras guardem uma relação inversa com a ocorrência de DCNT, essa associação não foi confirmada com os resultados da presente casuística. No entanto, as elevadas prevalências de excesso de peso e do baixo consumo de frutas, legumes e verduras, indicam a necessidade de intervenções, visando à implementação de estratégias de prevenção e promoção da saúde, incentivando a redução do excesso de peso e o maior consumo desses alimentos, ricos em fibras. 


\section{Colaboradores}

ECC Azevedo, FMRS Dias, AS Diniz e PC Cabral participaram igualmente de todas as etapas de elaboração do artigo.

\section{Referências}

1. World Health Organization (WHO). Diet, nutrition and prevention of chronic diseases. Report FAO/WHO Expert Consulation. Geneva: WHO; 2003. (WHO Technical Report Series 916)

2. Lerario DDG, Gimeno SG, Franco LJ, Iunes M, Ferreira SRG, Grupo de estudos em Diabetes na comunidade Nipo-brasileira. Excesso de peso e gordura abdominal para a síndrome metabólica em nipobrasileiros. Rev Saude Publica 2002; 36(1):4-11.

3. Instituto Brasileiro de Geografia e Estatística (IBGE). Pesquisa de Orçamento Familiar (POF) 2008- 2009: Antropometria e estado nutricional de crianças, adolescentes e adultos no Brasil. Rio de Janeiro: IBGE; 2010.

4. Brasil. Ministério da Saúde (MS). Secretaria de Vigilância em Saúde. Secretaria de Gestão Estratégica e Participativa. Vigitel Brasil 2010: vigilância de fatores de risco e proteção para doenças crônicas por inquérito telefônico. Brasília: MS; 2011.

5. Petribú MMV, Cabral PC, Arruda IKG. Estado Nutricional, Consumo alimentar e risco cardiovascular: Um estudo em universitários. Rev Nutrição 2009; 22(6):837-846.

6. Sartorelli DS, Franco LJ, Cardoso MA. High intake of fruits and vegetables predicts weight loss in Brazilian overweight adults. Nutr Res 2008; 28(4):233238.

7. Alves ALS, Olinto MTA, Costa JSD, Bairros FS, Balbinotti MAA. Padrões alimentares de mulheres adultas residentes em área urbana no Sul do Brasil. Rev Saude Publica 2006; 40(5):865-873.

8. Philipi ST, Latterza AR, Cruz ATR, Ribeiro LC. Pirâmide alimentar adaptada: guia para escolha dos alimentos. Rev Nutrição 1999; 12(1):65-80.

9. Furlan-viebig R, Pastor-valero M. Desenvolvimento de um questionário de freqüência alimentar para o estudo de dieta e doenças não transmissíveis. Rev Saude Publica 2003; 38(4):581-584.

10. Lohman TG, Roche AF, Martorell R. Anthropometric standardization reference manual. Toronto: Abridged; 1991.

11. World Health Organization (WHO). Physical status: The use and interpretation of anthropometry. Geneva: WHO; 1995. (WHO technical Report Series, 854).

12. World Health Organization (WHO). Obesity: preventing and managing the global epidemic. Geneva: WHO; 1998. (Report of a WHO Consultation on Obesity).

13. Heyward VH, Stolarcyk LM. Método de Impedância Bioelétrica. In: Heyward VH, Stolarcyk LM, organizadores. Avaliação da Composição Corporal Aplicada. São Paulo: Manole; 2000. p. 47-60.

14. Gallagher D, Heymsfield SB, Heo M, Jebb SA, Murgatroyd PR, Sakamoto Y. Healthy percentage body fat ranges: an approach for developing guidelines based on body mass index. Am J Clin Nutr 2000; 72(3):694-701.

15. Perozzo G, Olinto MTA, Dias-da-costa JS, Henn RL, Sarriera J, Pattussi MP. Associação dos padrões alimentares com obesidade geral e abdominal em mulheres residentes no Sul do Brasil. Cad Saude Publica 2008; 24(10):2427-2439. 
16. Whichelow MJ, Prevost AT. Dietary patters and their association with demographic, lifestyle and health variables in a radom sample of British adults. $\mathrm{Br} \mathrm{J}$ Nutr 1996; 76(1):17-30.

17. Gimeno SGA, Mondini L, Moraes SA, Freitas ICM. Padrões de consumo de alimentos e fatores associados em adultos de ribeirão Preto, São Paulo, Brasil: Projeto OBEDIARP. Cad Saude Publica 2011; 27(3): 533-545.

18. Departamento de Nutrição/UFPE, Instituto Materno Infantil de Pernambuco, Secretaria Estadual de Saúde. III Pesquisa Estadual de Saúde e Nutrição (PESN). Situação Alimentar, nutricional e de saúde no estado de Pernambuco: contexto socioeconômico e de serviços. Recife: Departamento de $\mathrm{Nu}-$ trição/UFPE, Instituto Materno Infantil de Pernambuco, Secretaria Estadual de Saúde; 2008.

19. Fornes NS, Martins IS, Velasquez-melendez G, Latorre MRDO. Escores de consumo alimentar e níveis lipêmicos em população de São Paulo, Brasil. Rev Saude Publica 2002; 36(1):12-18.

20. Castanho GKF, Marsola FC, Mclellan KCP, Nicola M, Moreto F, Burini RC. Consumo de frutas, verduras e legumes associado à Síndrome Metabólica e seus componentes em amostra populacional adulta. Cien Saude Colet 2013; 18(2):385-392.

21. Mondini L, Moraes AS, Freitas ICM, Gimeno SGA. Consumo de frutas e hortaliças por adultos em Ribeirão Preto, SP. Rev Saude Publica 2010; 44(4):686694.

22. Neutzling MB, Rombaldi AJ, Azevedo MR, Hallal PC. Fatores associados ao consumo de frutas, legumes e verduras em adultos de uma cidade no Sul do Brasil. Cad Saude Publica 2009; 25(11):2365-2374

23. Johnson F, Cooke L, Croker H, Wardle J. Changing perceptions of weight in Great Britain: comparison of two populations surveys. BMJ 2008; 337:a494.

24. Rodríguez-Rodríguez E, Aparicio A, López- Sobaler AM, Ortega RM. Percepción Del peso corporal y medidas adoptadas para su control em control em población española. Nutr. Hosp. 2009; 24(5):580-587.
25. Bernardi F, Cichelero C, Vitolo MR. Comportamento de restrição alimentar e obesidade. Revista de Nutrição 2005; 18(1):85-93.

26. Fett CA, Fett, WCR, Marchini JS, Ribeiro RPP. Estilo de vida e fatores associados ao aumento da gordura corporal de mulheres. Cien Saude Colet 2010; 15(1):131-140

27. Pinho CPS, Diniz AS, Arruda IKG, Lira PIC, Cabral PC, Siqueira LAS, Batista Filho M. Consumo de alimentos protetores e preditores do risco cardiovascular em adultos do estado de Pernambuco. Rev Nutr 2012; 25(3):341-351.

28. Sichieri R, Castro JFG, Moura AS. Fatores associados ao padrão de consumo alimentar da população brasileira urbana. Cad Saude Publica 2003; 19(Supl. 1):S47-53.

29. Lins APM, Sichieri R, Coutinho WF, Ramos EG, Peixoto MVM, Fonseca VM. Alimentação saudável, escolaridade e excesso de peso entre mulheres de baixa renda. Cien Saude Colet 2013; 18(2):357-366.

30. Hu FB. Dietary pattern analysis: a new direction in nutritional epidemiology. Curr Opin Lipidol 2002; 13(1):3-9.

31. Neumann AICP, Martins IS, Marcopito LF, Araujo EAC. Padrões alimentares associados a fatores de risco para doenças cardiovasculares entre residentes de um município brasileiro. Rev Panam Salud Publica 2007; 22(5):329-339.

Artigo apresentado em 09/05/2013

Aprovado em 25/01/2014

Versão final apresentada em 02/02/2014 